\title{
Thermal Stress During Separation for Ceramics Sleeve and Shaft Connected by Shrink Fitting*
}

\author{
Wenbin LI**, Hiromasa SAKAI**, Shota HARADA**, Yasushi TAKASE**, \\ Nao-Aki NODA** and Yoshikazu SANO** \\ **Department of Mechanical and Control Engineering, Kyushu Institute of Technology \\ Sensui-Cho 1-1 Tobata-Ku, Kitakyushu-Shi, Fukuoka, Japan \\ E-mail: noda@mech.kyutech.ac.jp
}

\begin{abstract}
Steel conveying rollers used in hot rolling mills must be exchanged frequently at great cost because hot conveyed strips induce wear and deterioration on the surface of roller in short periods. In this study, new roller structure is considered which has a ceramics sleeve connected with two short steel shafts at both ends by shrink fitting. Here, the ceramics sleeve may provide a longer life and reduce the cost for the maintenance. However, after used for a period the steel shaft has to be pulled out for exchange. Simply, heating outside surface and cooling inside surface of the shaft are necessary for separation. However, attention should be paid to the maximum thermal stress of the ceramics sleeve in the process of separation. In this paper, finite element method analysis is applied to the structure and thermal stress has been calculated with the varying dimensions of the structure. Also several effects on thermal stress have been investigated, such as the effect of shrink fitting ratio, outside diameter, the fitted length, thickness of shaft, materials and so on. Finally the most appropriate thermal conditions have been discussed to reduce maximum stress and make separation easy, which is very useful for designing of new rollers.
\end{abstract}

Key words: Contact Problem, Ceramics, Thermal Stress, Thermal Deformation, Finite Element Method

\section{Introduction}

Conveying rollers in hot rolling mills as shown Fig.1 are usually made of cast iron alloy, carbon steel or alloy steel. Since they are used under high temperature and corrosive atmosphere, wear and deterioration are easily induced on the surface in a short period. After the deterioration exceeds the critical value, it must be repaired for recycle.

In this study, the new roller structure is considered which has a ceramics sleeve ${ }^{(1)}$ and two short steel shafts connected by shrink fitting at both ends, as shown in Fig.2(d). Since the ceramics has high heat resistance and abrasion resistance, the exchanging cycle of roller can be extended in a large scale, and therefore, the reconstruction time and cost can be reduced. Moreover, the roller can be rotated easily and follow the speed of the transporting strips smoothly because of its light weight. In the previous study, for the structure of roller, the stress $\sigma_{\theta s}$ due to shrink fitting and the stress $\sigma_{\theta b}$ due to load distribution were analyzed separately ${ }^{(2)}$. In detail, the effects of shrink fitting ratio, length of fitted part, material of the sleeve, radius curvature on maximum stress $\sigma_{\theta \max }=\sigma_{\theta s}+\sigma_{\theta b}$ were also investigated. Besides, as shown in Fig.3, when the steel shaft is worn out, in order to

*Received 9 Nov., 2011 (No. R-11-0686) [DOI: 10.1299/jmmp.6.251]

Copyright $@ 2012$ by JSME 


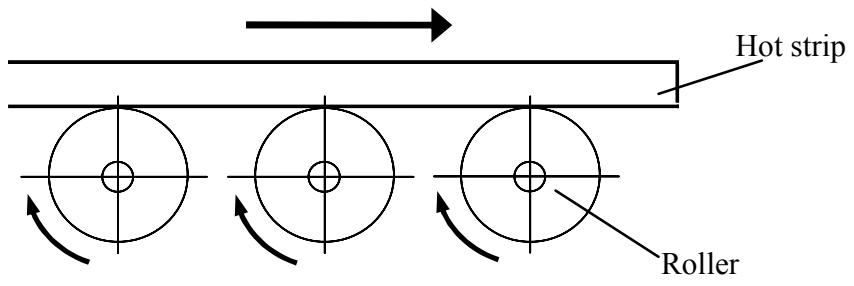

Fig.1 Layout of conveying rollers

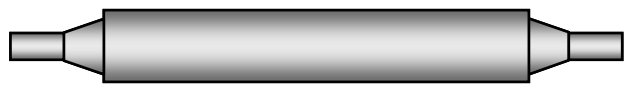

(a) Conventional roller

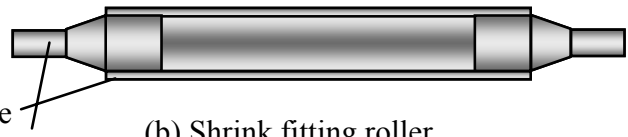

(b) Shrink fitting roller Shaft E

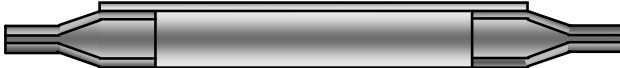

(c) New shrink fitting roller (Model A)

(d) New shrink fitting roller (Model B)

Fig.2 Roller structure

separate them, it is necessary to heat outside of the sleeve at fitted part and cool the inside of the shaft by water. Moreover, the effects of shrink fitting, outside diameter of the sleeve, thickness of the shaft, fitted length, conductivity of the sleeve on separation time are investigated $^{(3)}$.

In this study, therefore, the stress analysis for the structure model B shown in Fig.2(d) will be considered for investigation. The effects of shrink fitting, outside diameter of the sleeve, thickness of the shaft, fitted length, conductivity of the sleeve on thermal stress and maximum stress during separation are investigated by using the finite element method. Furthermore, in order to know the separation mechanism more accurately, simple double cylinder models are investigated and compared with real roller model ${ }^{(4)}$.

\section{Analytical Conditions of Real Roller}

\subsection{Boundary Conditions}

Define the shrink fitting ratio as $\delta / d$, where $\delta$ is the diameter difference with the diameter $d=210 \mathrm{~mm}$. Initially, the finite element method is applied to axisymmetric model B whose dimensions and boundary conditions are shown in Fig.3. The heating region is the outside surface of the contact part of sleeve as shown in Fig.3 (b). On the other hand, the water cooled region in Fig.3 (b) is the inside surface of the shaft, and the air cooled regions are the other regions including the left end surface of the shaft. In Fig.4, the assumed atmosphere temperature is shown according to the variation of a measured temperature in a furnace until the time 10000s. Then, forced convection as well as radiation is considered for heating. On the other hand, forced convection is assumed for water cooling, and natural convection for air cooling. First the temperature of roller was measured in the furnace. Then several values of heat transfer coefficient $\alpha$ and emissivity $\varepsilon$ were assumed and most suitable values were determined to produce almost the same the temperature in the experiment. Final values of $\alpha$ and $\varepsilon$ along the $\mathrm{r}, \mathrm{z}$ coordinate are shown in Table.1.

According to the symmetry of the problem, half model can be considered as shown in Fig.5. Therefore, along the surface $z=0$ insulation is applied as the thermal boundary condition, and $u_{z}=0, \tau_{r z}=0$ are applied as mechanical boundary conditions. Similarly, at the right end of the shaft, insulation and $\sigma_{z}=0, \tau_{r z}=0$ are assumed. Along the contact surface between the sleeve and shaft, heat transmission are actually caused by solid thermal 


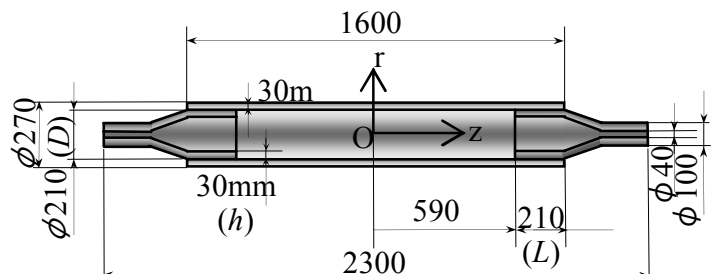

(a) Dimensions (mm)

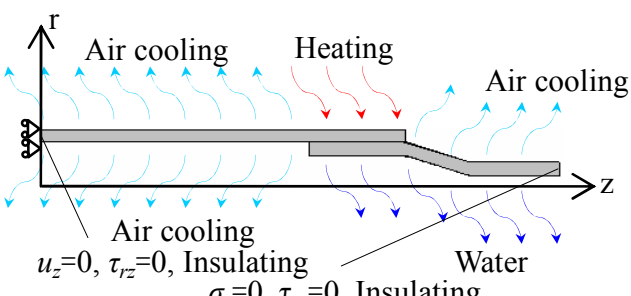

$\sigma_{z}=0, \tau_{\mathrm{rz}}=0$, Insulating

(b) Boundary condition

Fig.3 Model B initially considered

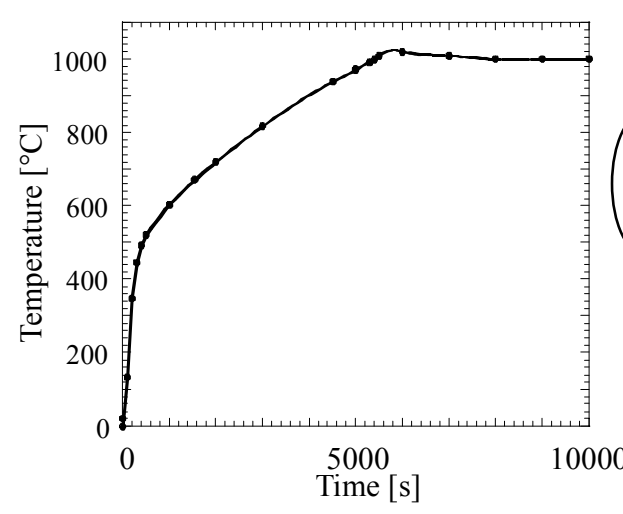

Fig.4 Atmosphere temperature

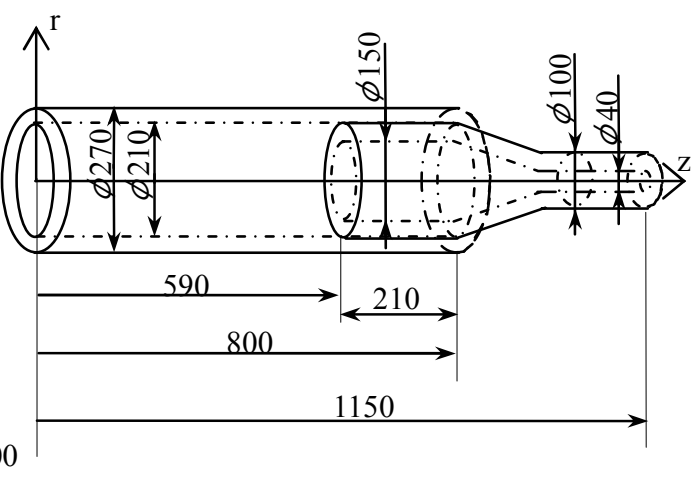

Fig.5 Real model B with coordinate $(\mathrm{mm})$

Table1 Values of heat transfer coefficient $\alpha$ and emissivity $\varepsilon$ along the r, z (mm) coordinate in Fig.5

\begin{tabular}{l|l}
\hline $\begin{array}{l}\text { Heat } \\
\text { (Forced convection, } \\
\text { Radiation) }\end{array}$ & $\begin{array}{l}\mathrm{r}=135, \mathrm{z}= \pm 590 \sim 800 \\
\varepsilon=0.4\end{array}$ \\
\hline Water cool & $\mathrm{r}=20, \mathrm{z}= \pm 590 \sim 1150$ \\
(Forced convection) & $\alpha=1.163 \times 10^{4}\left(\mathrm{~W} / \mathrm{m}^{2} \cdot \mathrm{K}\right)$ \\
& $\varepsilon=0$ \\
\hline & $\mathrm{r}=105, \mathrm{z}=0 \sim \pm 590$ \\
& $\mathrm{r}=135, \mathrm{z}=0 \sim \pm 590$ \\
& $\mathrm{r}=20 \sim 105, \mathrm{z}= \pm 590$ \\
& $\mathrm{r}=105 \sim 135, \mathrm{z}= \pm 800$ \\
Air cool & $\mathrm{r}=50 \sim 105, \mathrm{z}= \pm 800 \sim 1150$ \\
$($ Natural convection) & $(\mathrm{shaft} \operatorname{surface})$ \\
& $\alpha=50 \quad\left(\mathrm{~W} / \mathrm{m}^{2} \cdot \mathrm{K}\right)$ \\
& $\varepsilon=0$ \\
\hline \multirow{2}{*}{ Insulation } & $\mathrm{r}=105 \sim 135, \mathrm{z}=0$ \\
& $\mathrm{r}=20 \sim 50, \mathrm{z}= \pm 1150$ \\
\hline
\end{tabular}

conduction through real contact area, and thermal conduction through fluid lying in the space between the nominal contact surfaces ${ }^{(5)}$. Since the contact stress due to shrink fitting is quite large, solid thermal conduction seems to be predominant. In this analysis, therefore, contact heat transfer coefficient is assumed as $1.0 \times 10^{9} \mathrm{~W} / \mathrm{m}^{2} \cdot \mathrm{K}$. 
Table2 Material properties

\begin{tabular}{c|c|c|c}
\hline \hline & $\begin{array}{c}\text { Ceramics } \\
\mathrm{H}\end{array}$ & $\begin{array}{c}\text { Ceramics } \\
\mathrm{I}\end{array}$ & $\begin{array}{c}\text { Steel } \\
\left(H_{V} 200\right)\end{array}$ \\
\hline $\begin{array}{c}\text { Young's modulus } \\
{[\mathrm{GPa}]}\end{array}$ & 300 & 294 & 210 \\
\hline Poisson's ratio & 0.28 & 0.27 & 0.3 \\
\hline $\begin{array}{c}\text { Tensile strength } \\
{[\mathrm{MPa}]}\end{array}$ & 500 & 500 & 600 \\
\hline $\begin{array}{c}\text { Mass density } \\
{\left[\mathrm{kg} / \mathrm{m}^{3}\right]}\end{array}$ & 3200 & 3260 & 7800 \\
\hline $\begin{array}{c}\text { Thermal conductivity } \\
{[\mathrm{W} / \mathrm{m} \cdot \mathrm{K}]}\end{array}$ & $62.5(393 \mathrm{~K})$ & $17(393 \mathrm{~K})$ & 25 \\
\hline $\begin{array}{c}\text { Thermal expansion } \\
\text { coefficient }[1 / \mathrm{K}]\end{array}$ & $3.0 \times 10^{-6}$ & $3.0 \times 10^{-6}$ & $1.45 \times 10^{-5}$ \\
\hline $\begin{array}{c}\text { Specific heat } \\
{[\mathrm{J} / \mathrm{kg} \cdot \mathrm{K}]}\end{array}$ & 680 & 650 & 477 \\
\hline Emissivity & 0.4 & 0.4 & 0.4 \\
\hline
\end{tabular}

*: Thermal conductivity is linearly decreasing depending on temperature

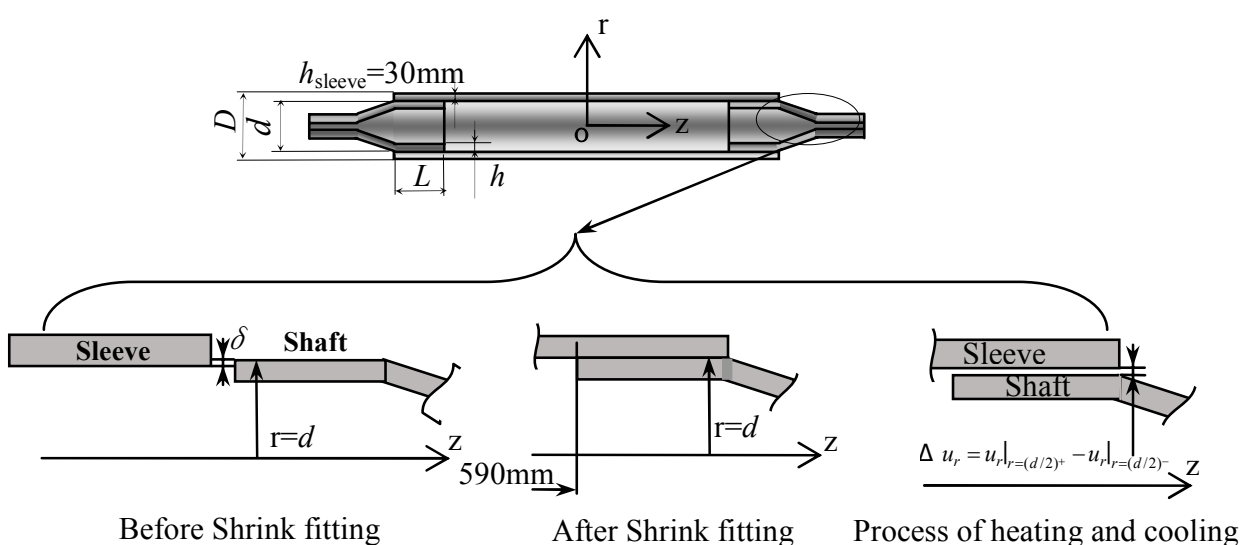

Fig.6 Relation of Dimensions and signs

\subsection{Material Properties}

Table 2 shows the material properties of the roller. In this study, the steel shaft is considered with Vickers hardness $H_{V}=200$. On the other hand, as the materials of the sleeve two kinds of ceramics $\mathrm{H}$ and ceramics I are considered compared with a steel sleeve with $\mathrm{H}_{\mathrm{v}}=200$. As shown in Table 2, it is assumed that the thermal conductivity of ceramics is linearly decreasing depending on the temperature. It has been told that the tensile strength of ceramics doesn't change below $1000^{\circ} \mathrm{C}^{(6)}$. It should be noted that the thermal expansion coefficient of steel is about 5 times as large as that of ceramics. It is also noted that under most cases of temperature the heat conductivity of ceramics $\mathrm{H}$ is about four times as large as that of ceramics I. 


\subsection{Analytical Model}

In this study, it is necessary to consider the thermal local deformations for sleeve and shaft in addition to heat transfer analysis for the whole structure. The thermal-mechanical coupled analysis is therefore performed for the models using quadrilateral axisymmetric element. Since it is known that the mechanical properties of ceramics does not change below $900^{\circ} \mathrm{C}$, elastic analysis is considered in this paper. As an example, the initial model B in Fig.3 (a) has 3760 elements and 4347 nodes. Axisymmetric model is used for analysis of half of the whole structure.

\section{Analytical Results for Real Roller}

\subsection{Maximum Stress and Temperature Distribution}

Here, the separation time is defined as the time when the displacement difference $\Delta u_{r}=\left.u_{r}\right|_{r=(d / 2)}-\left.u_{r}\right|_{v=(a / 2)}$ in the radial direction becomes larger than the initial radial difference $\delta / d$ along the contact region as shown in Fig.6. According to the analytical results, maximum stress of the sleeve is circumferential stress, appearing on the inside surface of sleeve. Figure 7 shows the temperature at the position where the maximum stress appears at 6246s. The temperature at the corresponding position on the outside surface of the sleeve is also shown in the whole process of separation for $\delta / d=0.3 \times 10^{-3}$. It should be noted that the sleeve and shaft are separated at $6615 \mathrm{~s}$. Before separation at $6615 \mathrm{~s}$, the temperature difference increases gradually while it becomes small after separation, which means the temperatures of inside and outside of the sleeve become almost same after separation. Figure 8 shows the temperature distribution along the inside and outside surface of the sleeve when the maximum stress appears at $6246 \mathrm{~s}$. It can be seen that temperature difference is smaller at separated area at two ends and larger at non-separated area in the middle area as shown from Fig. 8 because of the effect of water cooling. The temperature distribution of the sleeve is shown in Fig.9. Figure 10 shows the stress distribution along the $r$ direction from where the maximum stress appears. It can be seen that the stress becomes smaller from the inside to outside surface of the sleeve. Moreover, compressive stresses appear on the area heated early; while in this case, tensile stresses exist even on the outside surface of the sleeve which is heated with higher temperature. Figure 11 shows the stress distribution along the contact area of the sleeve when the maximum stress appears at 6246s. Stress distribution has two turning points at separated positions because of the temperature difference at separated positions as shown in Fig. $11^{(7)}$. It is thought that stress concentration appears at the end of contact part due to the temperature difference shown in Fig.8.

\subsection{Effect of Shrink Fitting Ratio on Maximum Stress}

Figure 12 shows the maximum stresses changing with time for different shrink fitting ratios. Here, the maximum stress is the stress $\sigma_{\theta s}$ due to shrink fitting before heated at $\mathrm{t}=0 \mathrm{~s}$. It can be seen that maximum stress is also large for large shrink fitting ratio. Moreover, maximum stress increases linearly with time to a peak value and decreases sharply. Maximum stress analysis is not necessary after separation. Stress distributions of $\sigma_{\theta \max }$ and $\sigma_{\theta s}$ when $\delta / d=0.3 \times 10^{-3}$ are shown in Fig.13. Moreover, the positions of $\sigma_{\theta s}=59.1 \mathrm{MPa}$ at $0 \mathrm{~s}$ and $\sigma_{\theta \max }=251.1 \mathrm{MPa}$ at $6246 \mathrm{~s}$ which is 4 times larger than $\sigma_{\theta s}$ 


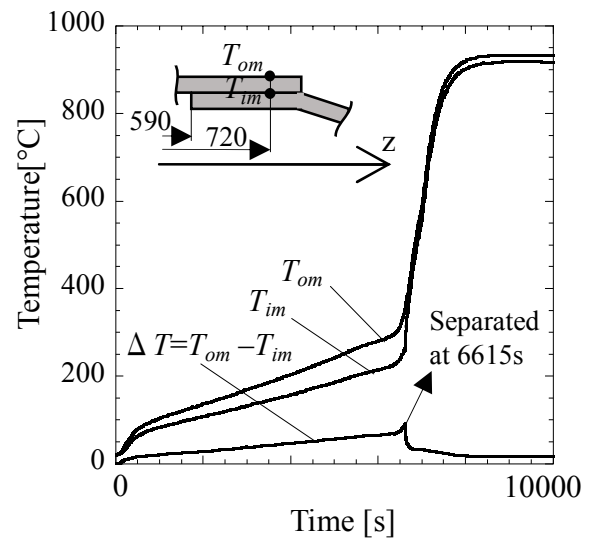

Fig.7 Temperature history in the whole process of separation

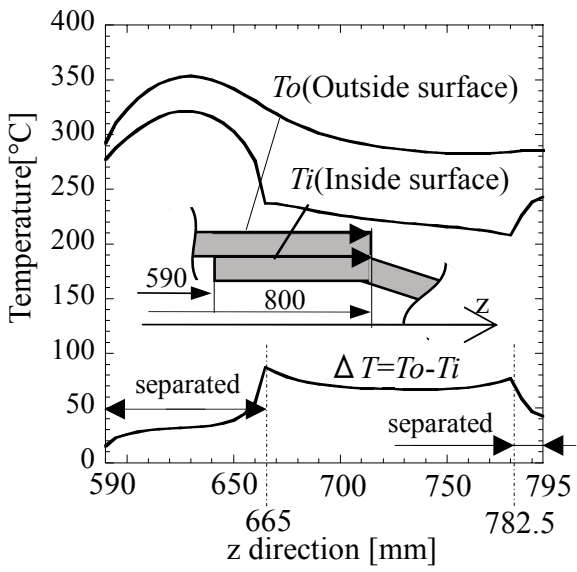

Fig.8 Temperature distribution along the interface when the maximum stress appears

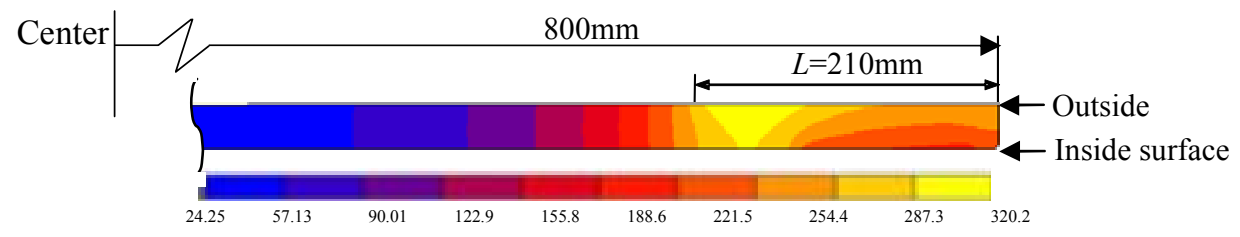

Fig.9 Temperature distribution of sleeve when maximum stress exists at $6246 \mathrm{~s}$ for $\delta / d=0.3 \times 10^{-3}$

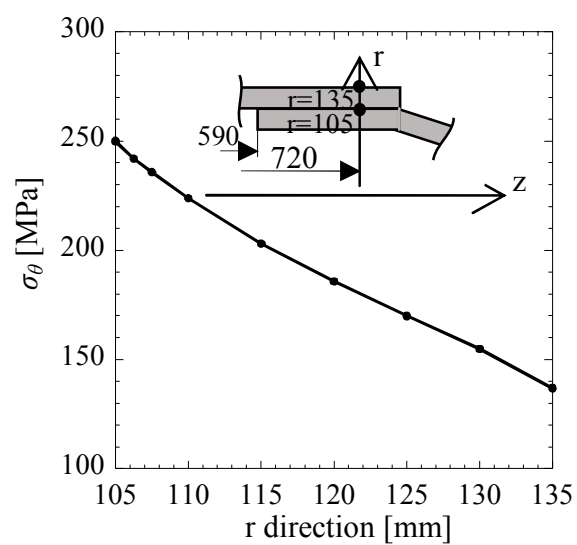

Fig.10 Stress distribution along $\mathrm{r}$ direction where and when maximum stress appears

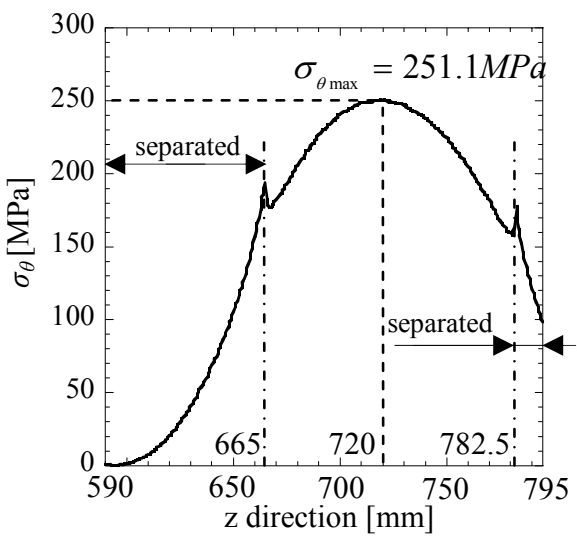

Fig.11 Stress distribution on the contact area when maximum stress appears

are also indicated in Fig.13. Figure 14 shows the relationship between shrink fitting ratio and separation time, while Fig.15 shows the relationship between shrink fitting ratio and $\sigma_{\theta \max }, \sigma_{\theta s}$. As shown in Figs.14, 15, it can be concluded that for the same shrink fitting ratio, maximum stress is large when separation time is long, which means heating time is long. Thus, as shrink fitting ratio increases, efficiency of separation will become low and maximum stress will increase, which results in the risk of destruction. Separation also becomes difficult. Therefore, the relationship between separation finishing time and maximum stress $\sigma_{\theta \max }$ will be investigated.

\subsection{Effect of Outside Diameter of Sleeve on Maximum Stress}

In the previous discussion, separation finishing time is long and maximum stress is large when shrink fitting ratio is large. Here, in order to discuss maximum stresses, the relationship between separation finishing time and maximum stress is investigated. Figure 


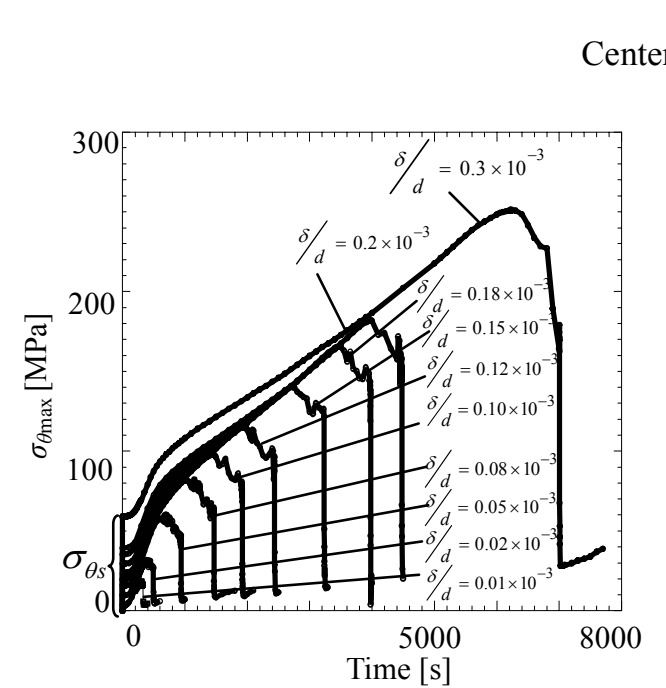

Fig.12 Maximum stresses vs. time for different $\delta / d$

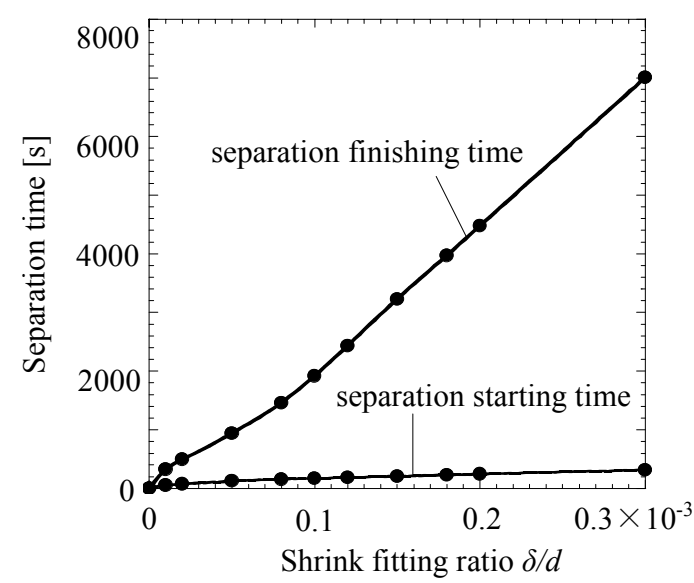

Fig.14 Effect of shrink fitting ratio on separation time

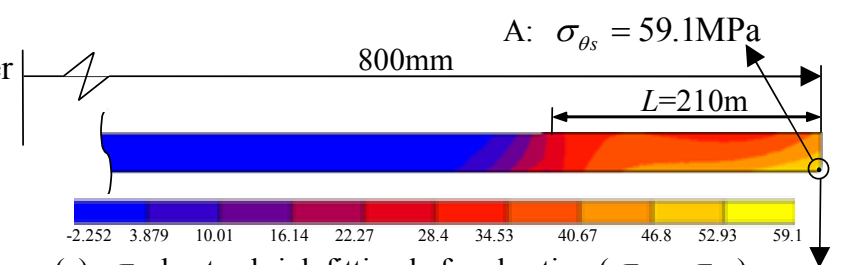

(a) $\sigma_{\theta}$ due to shrink fitting before heating $\left(\sigma_{\theta}=\sigma_{\theta s}\right.$ )
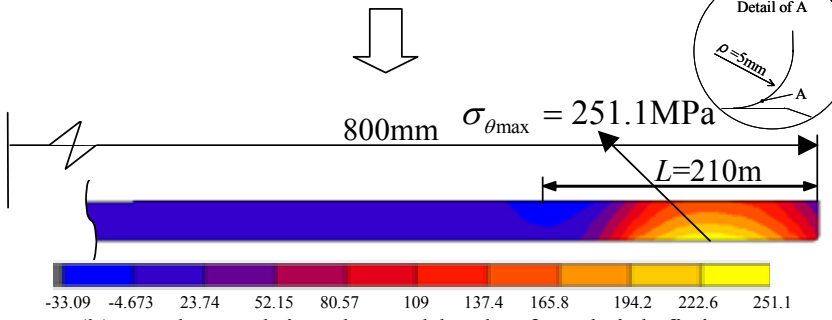

(b) $\sigma_{\theta}$ by applying thermal loads after shrink fitting when maximum stress appears

Fig.13 Stress distribution of sleeve $\left(\delta / d=0.3 \times 10^{-3}\right)$

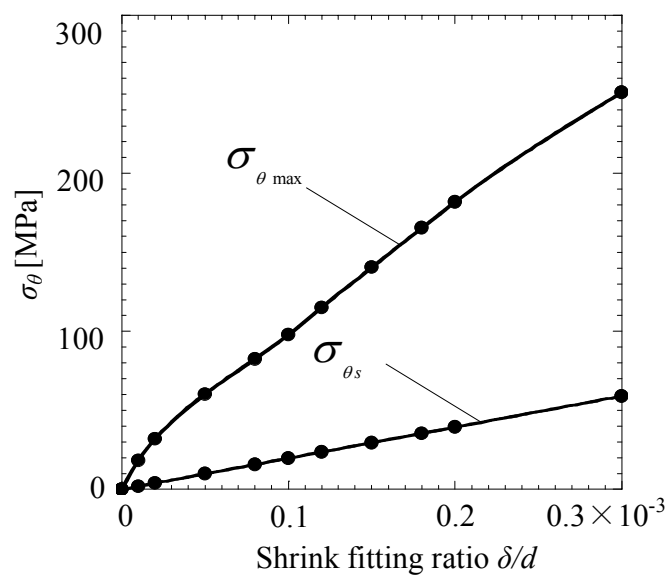

Fig.15 Effect of shrink fitting ratio on $\sigma_{\theta s}$ and $\sigma_{\theta \max }$

16 shows the relationship between separation finishing time and maximum stress for different outside diameters, $D=270 \mathrm{~mm}, 405 \mathrm{~mm}$ and $540 \mathrm{~mm}$. As shown in Fig 16 , when $\delta / d=0.3 \times 10^{-3}, \sigma_{\theta \max }=251.1 \mathrm{MPa}$ for $D=270 \mathrm{~mm}$ is about three times larger than $\sigma_{\theta \max }=87.34 \mathrm{MPa}$ for $D=540 \mathrm{~mm}$. As a result, a large $D$ can reduce the maximum stress for the same $\delta / d$. For different outside diameters, maximum stress changes at a same tendency with separation finishing time.

\subsection{Effect of Thickness $h$ of Shaft on Maximum Stress}

Figure 17 shows the relationship between separation finishing time and maximum stress for different thicknesses of shaft, $h=20 \mathrm{~mm}, 30 \mathrm{~mm}$ and $40 \mathrm{~mm}$. Here, for $h=40 \mathrm{~mm}$, separation can't be realized completely when $\delta / d>0.08 \times 10^{-3}$; so the results of $\delta / d \leq 0.08 \times 10^{-3}$ are shown for $\mathrm{h}=40 \mathrm{~mm}$. In addition, the maximum stress has a very large value $\sigma_{\theta \max }=633.1 \mathrm{MPa}>500 \mathrm{MPa}$ for $h=40 \mathrm{~mm}$ when $\delta / d=0.08 \times 10^{-3}$ as shown in 


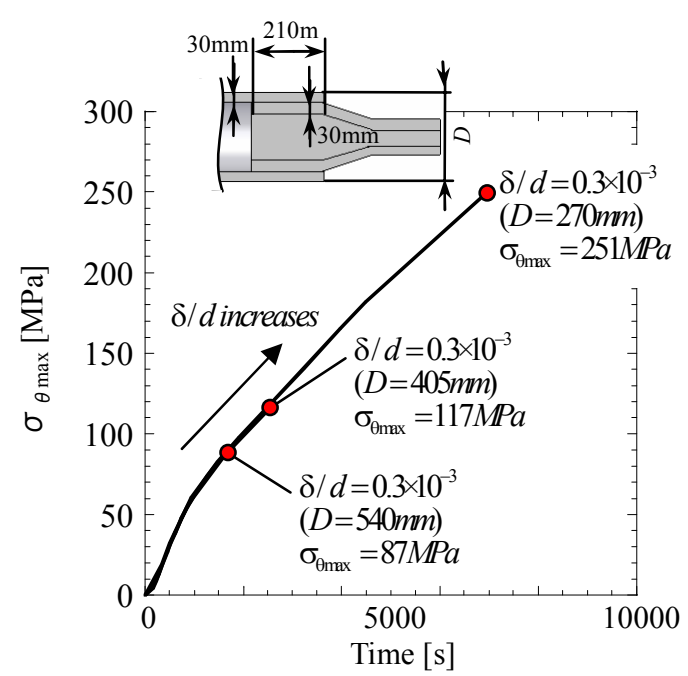

Fig.16 Effect of $D$ on $\sigma_{\theta \max }$

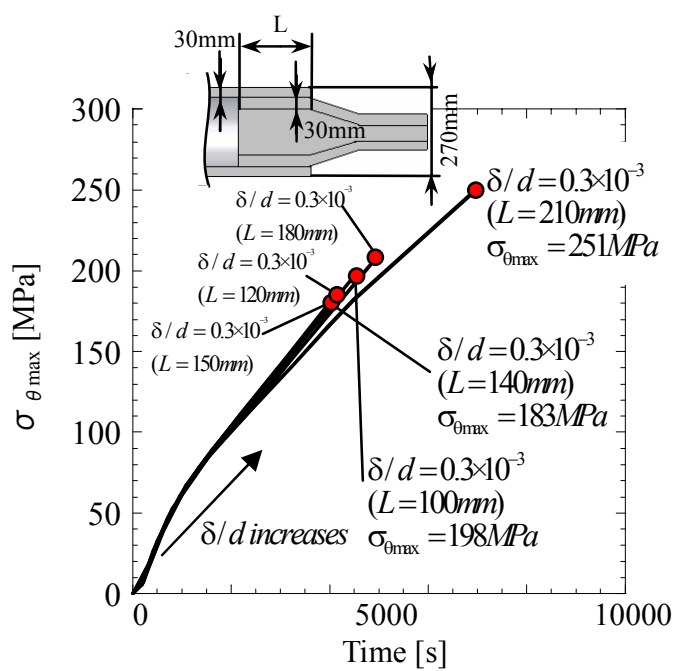

Fig. 18 Effect of $L$ on $\sigma_{\theta \max }$

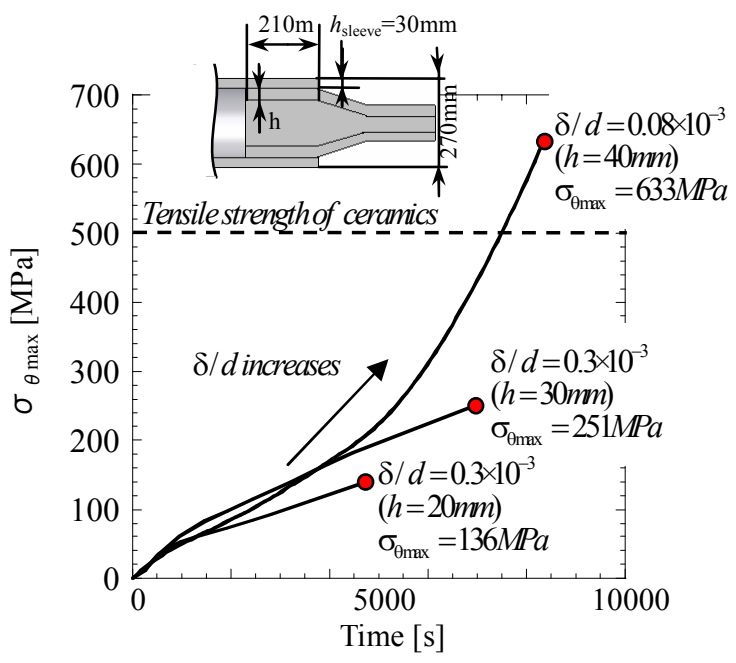

Fig.17 Effect of $h$ on $\sigma_{\theta \max }$

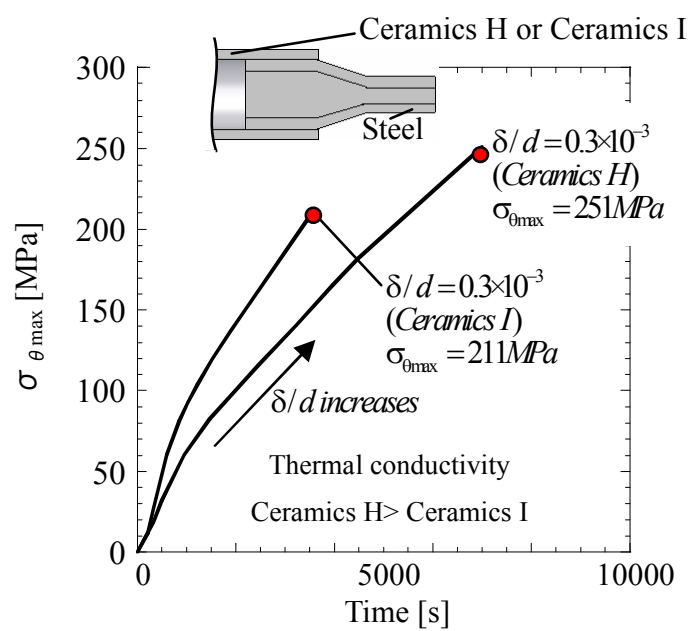

Fig.19 Effect of thermal conductivity on $\sigma_{\theta \max }$

Fig17. When $\delta / d=0.3 \times 10^{-3}$, maximum stress $\sigma_{\theta \max }=251.1 \mathrm{MPa}$ for $h=30 \mathrm{~mm}$ is about twice as large as $\sigma_{\theta \max }=136.9 \mathrm{MPa}$ for $h=20 \mathrm{~mm}$. As a result, small $h$ can reduce the maximum stress for the same $\delta / d$. For different thicknesses of shaft, maximum stress changes at a same tendency with separation finishing time.

\subsection{Effect of Fitted Length $L$ on Maximum Stress}

Usually, the fitted length $L$ is designed to have a proportion to the inside diameter of the sleeve. If the fitted length is changed, the water cooing region is also changed, which influences maximum stress. Here, six fitted lengths are considered for investigation, $L=100 \mathrm{~mm}, L=120 \mathrm{~mm}, L=140 \mathrm{~mm}, L=150 \mathrm{~mm}, L=180 \mathrm{~mm}$ and the initial $L=210 \mathrm{~mm}$. In order to check the effect, the length of heating part is fixed as a constant value of $210 \mathrm{~mm}$, while water cooled part and air cooled part can be changed with the fitted length.

Figure 18 shows the relationship between separation finishing time and maximum stress for six different fitted lengths. When $\delta / d=0.3 \times 10^{-3}$, maximum value of 
$\sigma_{\theta \max }=251.1 \mathrm{MPa}$ appears for $L=210 \mathrm{~mm}$ and minimum value of $\sigma_{\theta \max }=183.5 \mathrm{MPa}$ appears for $L=140 \mathrm{~mm}$. Generally, small $L$ can reduce separation time and maximum stress. However, maximum stress becomes a little larger when $L$ becomes shorter than $L=140 \mathrm{~mm}$. The separation time becomes longer, which is because the effect of water cooled is also too small if $L$ is too short. Similarly, maximum stress changes at a same tendency with separation finishing time.

\subsection{Effect of Thermal Conductivity of Sleeve on Maximum Stress}

In this study, two kinds of ceramics in Table 2 are considered for the sleeve. Figure 19 shows the relationship between separation finishing time and maximum stress for two kinds of ceramics sleeve. As shown in Fig.19, maximum stress for ceramics I is smaller than that for ceramics $H$. As a result, small thermal conductivity of sleeve can reduce maximum stress. Similarly, maximum stress changes at a same tendency with separation finishing time.

\section{Analytical Conditions of Double Cylinder Models}

\subsection{Double Cylinder Model}

Separation mechanism has been discussed for the real rollers in the previous sections. However, separation mechanism may change according to the boundary conditions. In order to know the separation mechanism more accurately, simple double cylinder models connected by shrink fitting are investigated, which could be compared with the real roller model.

In this study, the fitted part of roller model is considered as double cylinder model for investigation. Separation mechanism and maximum stress are analyzed by adding different boundary conditions to double cylinder models.

\subsection{Boundary Conditions}

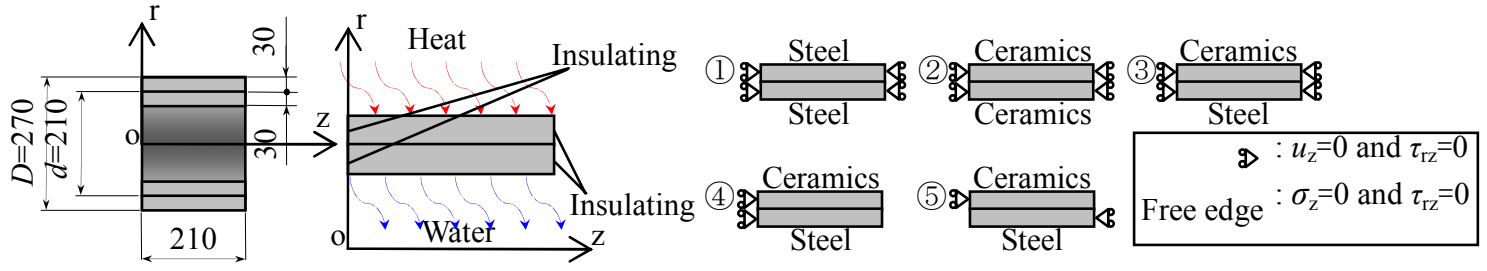

$\begin{array}{lll}\text { (a) Dimensions (mm) } & \text { (b) Thermal Boundary condition }\end{array}$

(c) Mechanical Boundary condition

Fig.20 Five double cylinder models

Table3 Values of heat transfer coefficient $\alpha$ and emissivity $\varepsilon$ along the $\mathrm{r}, \mathrm{z}(\mathrm{mm})$ coordinate in Fig.21

\begin{tabular}{l|l}
\hline \hline $\begin{array}{r}\text { Heat } \\
\text { Rorced convection, } \\
\text { Radiation) }\end{array}$ & $\begin{array}{l}\mathrm{r}=135, \mathrm{z}= \pm 0 \sim 210 \\
\alpha=50\left(\mathrm{~W} / \mathrm{m}^{2} \cdot \mathrm{K}\right)\end{array}$ \\
\hline & $\mathrm{r}=105, \mathrm{z}= \pm 0 \sim 210$ \\
Water cool & $\alpha=1.163 \times 10^{4}\left(\mathrm{~W} / \mathrm{m}^{2} \cdot \mathrm{K}\right)$ \\
(Forced convection) & \\
\hline Insulation & $\mathrm{r}=0.4$ \\
\hline
\end{tabular}

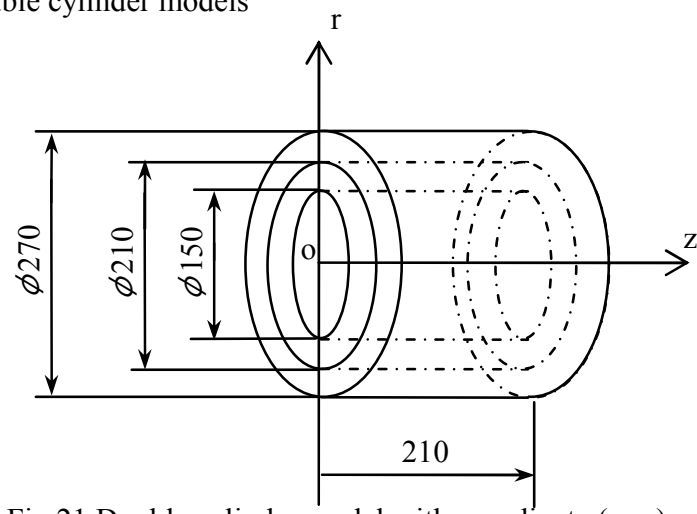

Fig.21 Double cylinder model with coordinate $(\mathrm{mm})$ 
Dimensions and boundary conditions are shown in Fig.20. Dimensions and thermal boundary conditions remain unchanged, while materials of double cylinders and fixed types at two ends are changed for investigation. Heating time is assumed as 10,000. As shown in Fig.20(b), outside surface of outside cylinder is heated and inside surface of shaft is cooled by water. Besides, atmosphere temperature in Fig. 4 is used as heating temperature. Heat transfer coefficient and emissivity for convection and radiation along the $r, z$ axis are shown in table 3 .

Moreover, similarly with the roller model, solid thermal conduction is predominant and contact heat transfer coefficient is assumed as $1.0 \times 10^{9} \mathrm{~W} / \mathrm{m}^{2} \cdot \mathrm{K}$.

\subsection{Material Properties}

Material properties are shown in table2. For double cylinder model, ceramics $\mathrm{H}$ is used for outside cylinder and steel for inside cylinder.

\subsection{Analytical Model}

In this study, same with roller model, thermal-mechanical coupled analysis is performed using quadrilateral axisymmetric element. There are 2016 elements and 2210 nodes in double cylinder models. Axisymmetric model is used for analysis of half of the whole structure.

\section{Results of Double Cylinder Models}

\subsection{Separation Mechanism for Double Cylinder Model 1 to Model 3}

Two ends are fixed for model 1, 2 and 3. Separation finishing times for different shrink fitting ratios are shown in table4 for model 1, 2 and 3. For model 1, inside and outside cylinder are both steel with a large linear expansion. Even when shrink fitting ratio is a large value $\delta / d=0.3 \times 10^{-3}$, separation can be realized; for model 2 , both inside and outside are ceramics with a small linear expansion. Separation can be realized for small shrink fitting ratios; for model 3, outside is ceramics $\mathrm{H}$ and inside is steel, which means inside can expand more than outside with a same temperature difference so that separation becomes more difficult. Even when $\delta / d=0.1 \times 10^{-3}$, separation can't be realized.

\subsection{Separation Mechanism of Model 4, 5 and Roller model}

Figure 22 shows the displacement difference in the $r$ direction along the contact surface between outside and inside cylinder when separation starts, finishes and in the process of separation. For the model whose outside is ceramics and inside is steel, separation can't be realized; however, separation can be realized for model 4 and 5 after the boundary conditions are changed.

In Fig.23, the displacements in $r$ direction along the contact surface between outside and inside are shown for model 4, 5 and the roller model. As shown in Fig.23(a),(b), separation starts from the right end for model 4 while starts from the left end for model 5 . It is thought that separation is different for model 4 and 5 because of the different fixed conditions. For example, the thermal deformation is shown in Fig. 24 when a simple steel cylinder is heated on the outside surface and the left end is fixed. When the left end is fixed, displacement of outside surface is larger than that of inside surface at the right end in the $r$ 
Table 4 Separation finishing time (mode1, 2, 3)

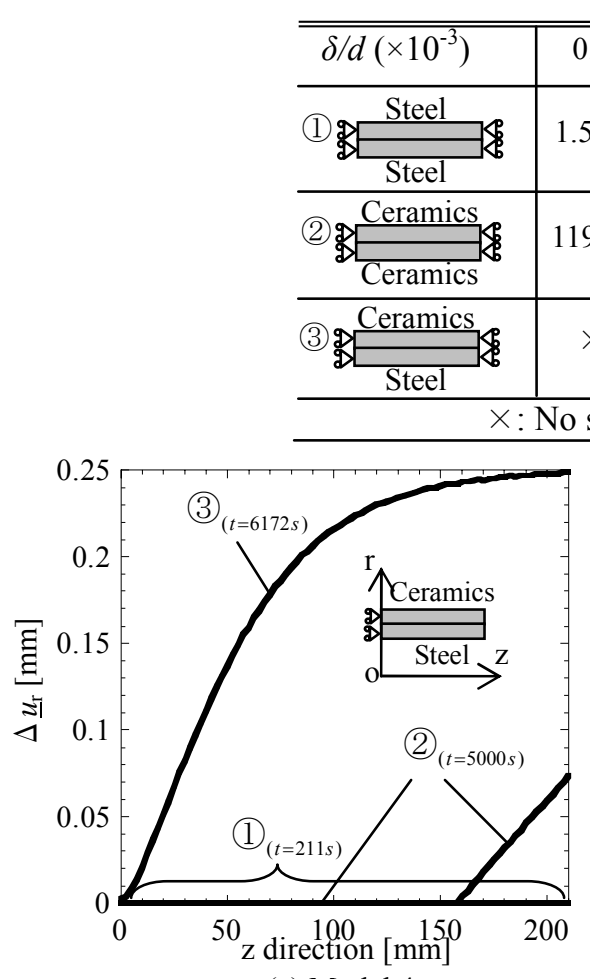

(a) Model 4

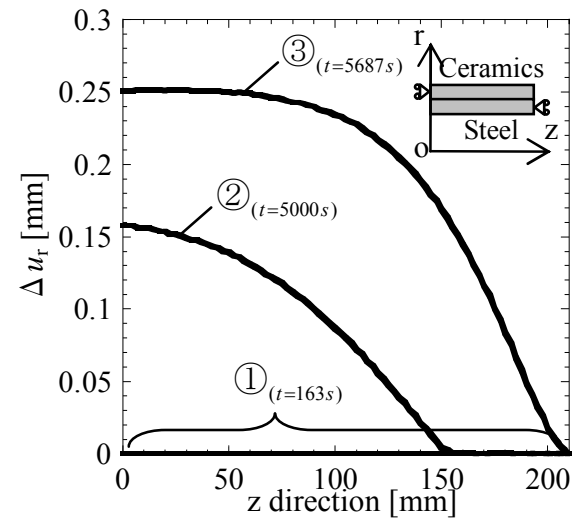

(b) Model 5

Fig.22 $\Delta u_{\mathrm{r}}$ vs. Contact area for five double cylinder model $4,5\left(\delta / d=0.3 \times 10^{-5}\right)$ (Time progress: (1) $\rightarrow$ (2) $\rightarrow$ (3)

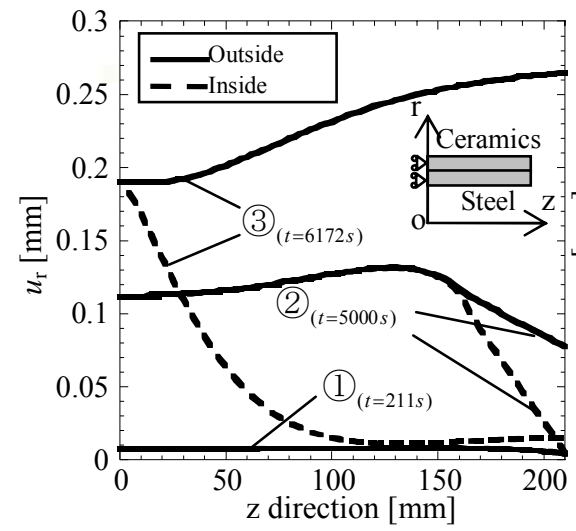

(a) Model 4

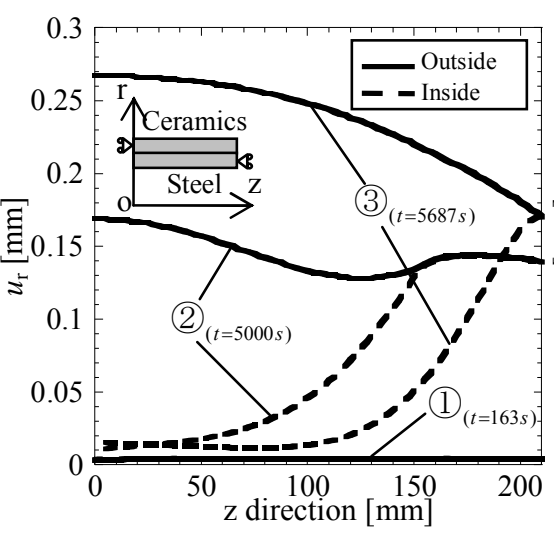

(b) Model 5

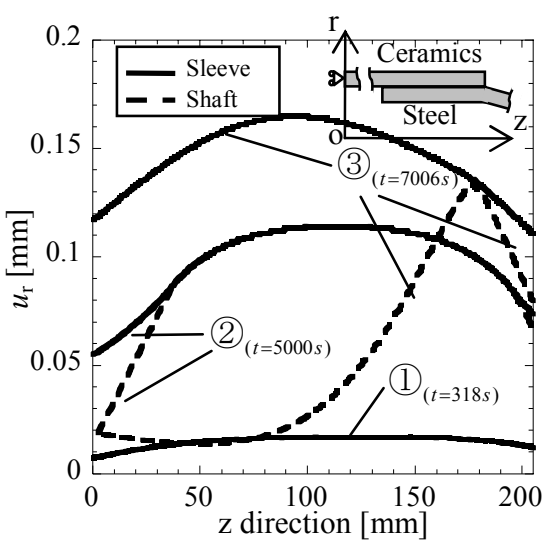

(c) Real model B

Fig.23 $u_{\mathrm{r}}$ vs. Contact area for double cylinder model 4,5 and Model B $\left(\delta / d=0.3 \times 10^{-5}\right)$ (Time progress: (1) $\rightarrow$ (2) $\rightarrow$ (3)

direction due to expansion as shown in Fig.24. Similarly to Fig.25, the thermal deformation is given for double cylinder model 4 and 5 . The left ends of outside cylinder are both fixed for model 4 and 5, so the displacement at the right end in the r direction is large. However, the left end of inside cylinder is also fixed for model 4 while the right end of inside cylinder is fixed for model 5. Therefore, the displacement in the $r$ direction becomes larger at right end for model and left end for model 5. The displacement difference has a maximum value at right end and separation starts from the left end for model 5. For model 4, separation starts from the right end. The displacement of outside and inside cylinder is also large at the right end; however, because the linear expansion coefficient of steel inside is larger than ceramics outside, the displacement difference is induced more difficultly than model 5. Therefore, separation time is longer than model 5. Moreover, as shown in Fig.23(c), for roller model, 


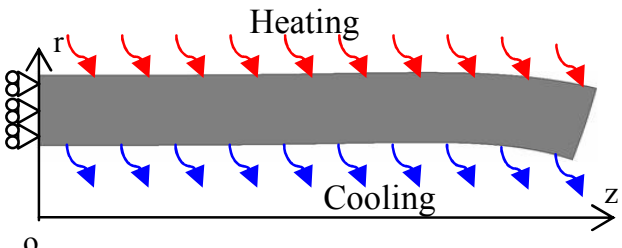

Fig.24 Thermal deformation of steel single cylinder model

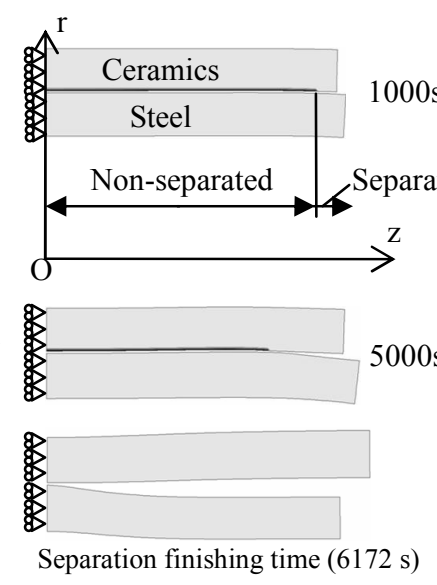

(a) Model 4

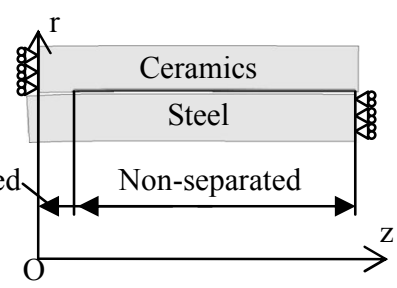

$\$$
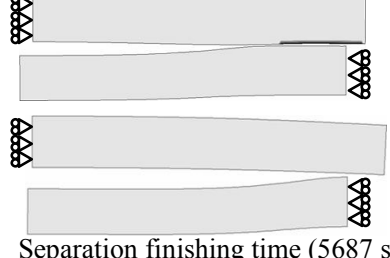

(b) Model 5

Fig.25 Thermal deformation of double cylinder model $4,5\left(\delta / d=0.3 \times 10^{-3}\right)$ (magnification of displacement: $\mathrm{mm} \times 50$ )

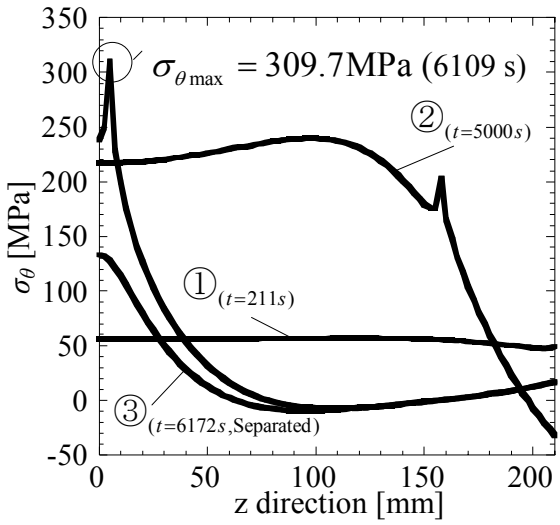

(a) Model 4

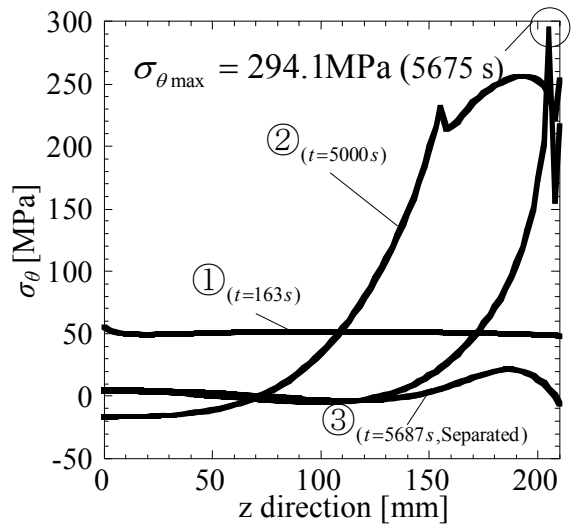

(a) Model 5

Fig.26 Stress distribution along $\mathrm{z}$ direction of model $4,5\left(\delta / d=0.3 \times 10^{-3}\right)$

(Time progress: (1) $\rightarrow$ (2) $\rightarrow$ (3)

separation starts from the left end because the boundary conditions are similar with model 5 . In addition, separation is also induced from the right end and starting from the left end later after separation.

\subsection{Stress Analysis for Double Cylinder Model 4 and 5}

Figure 26 shows stress distribution along the inside surface of outside cylinder at different separation times. Similarly with the results of roller model as shown in Fig.11, maximum stress appears at the position where separation has just happened. Moreover, maximum stress appears just a little before separation happens for model 4, 5 and roller model. Stress of the whole structure decreases sharply after separation is completely induced.

\section{Conclusions}

In this paper, ceramics conveying rollers with ceramics sleeves and steel shafts of which inside is hollow are considered for investigation. In order to exchange the shafts, 
heating outside surface of sleeve and cooling inside surface of shaft are applied. Thermal stress has been investigated during separation. Here, separation is considered to be finished when all the fitted part separates. Therefore, different dimensions, shapes, material properties and their effects have been discussed, which are shown in the following.

(1) Maximum stress of the sleeve is circumferential stress, appearing on the inside surface of sleeve. Maximum stress doesn't appear when temperature difference is largest, which appears a little bit before separation happens.

(2) Maximum stress becomes larger and separation finishing time becomes longer with shrink fitting ratio increasing. It is thought that the effect increases which is induced by the increasing temperature of steel shafts with a large linear expansion coefficient when shrink fitting ratio becomes larger.

(3) Large outside diameter of sleeve can reduce the maximum stress for the same shrink fitting ratio. When $\delta / d=0.3 \times 10^{-3}$, maximum stress for $D=540 \mathrm{~mm}$ is about $1 / 3$ of that for $D=270 \mathrm{~mm}$ (see Fig.16).

(4) Small thickness of shaft at fitted part can reduce the maximum stress for the same shrink fitting ratio. When $\delta / d=0.3 \times 10^{-3}$, maximum stress for $h=20 \mathrm{~mm}$ is about $1 / 2$ of that for $h=30 \mathrm{~mm}$ (see Fig.17). For $h=40 \mathrm{~mm}$, separation can't be realized when $\delta / d \geq 0.08 \times 10^{-3}$ and maximum stress exceeds the tensile strength.

(5) When fitted length decreases, maximum stress becomes smaller for the same shrink fitting ratio. However, when $\delta / d=0.3 \times 10^{-3}$, maximum stress has a minimum value for $L=140 \mathrm{~mm}$ and maximum stress increases again if fitted length becomes small (see Fig.18).

(6) Material of sleeve with a small thermal conductivity can reduce maximum stress (see Fig.19).

(7) If shrink fitting ratio becomes smaller, outside diameter of sleeve becomes larger, thickness of shaft becomes smaller or thermal conductivity of ceramics sleeve becomes smaller, maximum stress can be reduced, which coincides with that separation time becomes shorter.

(8) For double cylinder models whose two ends are both fixed, separation can be realized when outside and inside are both steel with a large linear expansion coefficient; separation becomes difficult if outside is ceramics with a small linear expansion coefficient.

(9) It is possible for separation to be realized when both outside and inside are ceramics with a small linear expansion coefficient. However, separation time is longer than that when both outside and inside are steel.

\section{Reference}

(1) Iwata, T. and Mori, H., "Material Choice for Hot Run Table Roller", Plant Engineer, Vol.15, No.6 (1983), pp.55-59 (in Japanese).

(2) Masakazu Tsuyunaru, Nao-Aki Noda, Hendra, and Yasushi Takase, "Maximum Stress for Shrink Fitting System Used for Ceramics Conveying Rollers", Transactions of the Japan Society of Mechanical Engineering, Vol.74, No.743 (2008), pp.919-925.

(3) Wenbin Li, Hiromasa Sakai, Yasushi Takasei, Nao-Aki NODA, "Analysis of Separation Conditions for Shrink Fitting System Used for Ceramics Sleeve", Journal of Solid Mechanism and Materials Engineering, Vol5, No.1(2011), pp14-24.

(4) Wenbin Li, Hiromasa Sakai, Shota Harada, Yasushi Takase, Nao-Aki Noda, "Separation Mechanism for Double Cylinder With Shrink Fitting System Used for Ceramics 
Conveying Rollers", Frontiers of Mechanical Engineering, 6(3)(2011), pp.277-286

(5) Torii, K., "Heat Transfer Across the Solid Interface Governed by its Microscopic Surface-Structure -Interface between Macro-and Micro-Mechanics", The Japan Society of Mechanical Engineers, Vol.96, No.892 (1993), pp.198-203 (in Japanese) .

(6) The 136th Committee on Future-Oriented Machining of Japan Society of the Promotion Science, Technology Handbook on Fine Ceramics, (1998), pp.30-31, 67, 82, 414-419 (in Japanese), Uchida Rokakuho.

(7) S, Timoshenko, Strength of Materials, Part II (1976), pp.212, Robert.E Krieger Publishing Company. 